Research Article

\section{Effect of laparoscopic salpingectomy on subsequent ovarian response after controlled ovarian hyperstimulation}

\author{
Chadi Yazbeck ${ }^{1,2 *}$, Asma Boukadida², Christian Chauvin ${ }^{3}$, Anne \\ Laure Margulies ${ }^{1}$ and Ségolène Falcone ${ }^{3}$ \\ 'Department of Obstetrics, Gynecology and Reproductive Medicine, CMC Hartmann Cherest \\ Clinics, 26 Bd Victor Hugo 92200 Neuilly Sur Seine, France \\ ${ }^{2}$ Department of Obstetrics, Gynecology and Reproductive Medicine, Foch University Hospital, 40 \\ rue Worth, 92150 Suresnes, France \\ ${ }^{3}$ Department of Obstetrics, Gynecology and Reproductive Medicine, Bichat Claude Bernard \\ University Hospital, APHP, 75018 Paris, France
}

\section{Abstract}

Background: The effect of salpingectomy on ovarian response is a matter of debate. Due to conflicting data, alternative techniques were developed to perform salpingectomy for treatment of hydrosalpinges in infertile patients. This study aims to evaluate the effect of salpingectomy on ovarian response after stimulation with gonadotropins.

Methods: In a retrospective analysis, one hundred fifty-seven patients with tubal infertility were divided into three groups according to their surgical histories: bilateral salpingectomy (BS group); unilateral salpingectomy (US group); and no history of salpingectomy (NS group). Ovarian response and IVF outcomes were compared between groups by analysis of variance. Prognostic factors for ovarian response were estimated by linear regression models.

Results: In the BS group, the total numbers of oocytes retrieved, and embryos obtained were significantly lower than those in the NS group $(p=0.02)$. Poor ovarian response was also more frequent in the BS group $(p=0.02)$. In the US group, follicle development was reduced on the operated side. This effect was more pronounced when salpingectomies were performed for hydrosalpinges than when performed for ectopic pregnancies, and significant decreases were observed in follicle recruitment $(p=0.005)$ and oocyte retrieval $(p=0.02)$ on the operated side.

Conclusion: Salpingectomy could have a minor negative effect on ovarian response. This is particularly true with bilateral salpingectomies, in which the ovarian blood supply could be disrupted, with no possible compensation by the contralateral side.

\section{More Information}

*Address for Correspondence: Chadi Yazbeck, MD, MPH, PhD, Obstetrics, Gynecology and Reproductive Medicine Department, 60 avenue Paul Doumer, 75016 Paris, France, Tel.: +33 145 0390 90; Fax: +331450303 32; Email: contact@dryazbeck.fr

Submitted: 01 September 2019 Approved: 17 September 2019 Published: 18 September 2019

How to cite this article: Yazbeck C, Boukadida A, Chauvin C, Margulies AL, Falcone S. Effect of laparoscopic salpingectomy on subsequent ovarian response after controlled ovarian hyperstimulation. Clin J Obstet Gynaecol. 2019; 2: 107-112.

DOI: dx.doi.org/10.29328/journal.cjog. 1001030 Copyright: @ 2019 Yazbeck C, et al. This is an open access article distributed under the Creative Commons Attribution License, which permits unrestricted use, distribution, and reproduction in any medium, provided the original work is properly cited

Keywords: Assisted reproductive technology; Fallopian tubes; Ovarian reserve; Reproductive surgery; Salpingectomy

Check for updates

\section{Introduction}

Salpingectomy is a common operation, performed for the management of ectopic pregnancy (EP) and, beginning approximately fifteen years ago, in women with hydrosalpinges undergoing in vitro fertilization (IVF) [1]. In fact, several reports have indicated that the presence of hydrosalpinges has a detrimental effect on implantation and pregnancy rates [2-9]. Prophylactic salpingectomy in women with large hydrosalpinges has been shown to be beneficial in terms of increased pregnancy and live birth rates after IVF treatment [10-12].

However, salpingectomy is suspected to disrupt ovarian function and to decrease ovarian response to the gonadotropins used in assisted reproductive technology (ART), by reducing the vascular supply to the ovary [13]. While some authors found substantial effects of salpingectomy on ovarian response [13-16], others have not [17-22].

Given these conflicting data and the emergence of alternative techniques to perform salpingectomy for treatment of hydrosalpinges in infertile patients, the question of the effect of salpingectomy on ovarian function is more seriously debated than ever before. This study aimed to investigate the effects of salpingectomy on ovarian response to stimulation and its consequences on IVF outcomes. 


\section{Materials and Methods}

\section{Patient selection}

One hundred fifty-seven patients with tubal infertility, admitted to the IVF center of Bichat Claude Bernard University Hospital (Paris), were enrolled in a prospective cohort study. Inclusion criteria were female patient's age below 40 years at enrollment and tubal infertility as the main etiology indicating standard IVF. Exclusion criteria involved surgical history of oophorectomy or ovarian cystectomy, polycystic ovaries, uterine malformation, and severe male infertility (i.e azoospermia).

Before IVF treatment, all the couples underwent routine fertility screening, in particular a hysterosalpingography, an antral follicle count by pelvic ultrasonography and baseline hormonal tests for ovarian reserve. Data from previous reports of surgical interventions were recorded. The mean follow-up period was 24 months.

Among the 157 patients included, 66 patients had a history of salpingectomy, including bilateral (BS group) and unilateral (US group) salpingectomy, done mainly by reproductive surgeons of the ob-gyn department. Ninety-one patients with no history of salpingectomy or hydrosalpinges were set as controls (NS group).

\section{Ovarian stimulation}

All patients were stimulated using one of our standard GnRH agonist or antagonist protocols. Starting recombinant FSH dose was set at $225 \pm 75$ IU according to patients' age and baseline ovarian reserve. Recombinant human chorionic gonadotropin (rhCG) trigger was administered when at least three mature follicles (more than $17 \mathrm{~mm}$ in diameter) were obtained. Follicles between 10 and $16 \mathrm{~mm}$ and those equal to or greater than $17 \mathrm{~mm}$ were counted in each ovary by ultrasonography on the rhCG day.

Oocyte retrieval by ultrasound-guided aspiration was performed by the same reproductive surgeons, thirty-six hours after rHCG, and the number of oocytes retrieved from each ovary was stippled. Insemination or microinjection was performed immediately after, using classical techniques of IVF or ICSI. Forty-eight hours after insemination or microinjection, embryo transfer was performed upon analysis of embryo quality. Progesterone was administered at $400 \mathrm{mg}$ per day intravaginally for luteal phase support.

Poor ovarian response was defined as total oocyte retrieval equal to or less than three. Implantation rate was defined as the number of gestational sacs per embryo transferred per patient. Clinical pregnancy was defined as the presence of an intrauterine gestational sac with fetal cardiac activity at the five-week pelvic ultrasound.

\section{Outcomes}

The primary outcome measurement was the total number of oocytes retrieved per cycle. The secondary outcome measurements were the number of follicles recruited between 10 and $16 \mathrm{~mm}$ in size and those larger than $16 \mathrm{~mm}$, the peak estradiol level on the day of rHCG, poor ovarian response, number of embryos obtained and clinical pregnancy rate per cycle.

\section{Statistical analysis}

Data analysis was performed using SAS software, version 9.1.3 (SAS Institute, Cary, NC, USA). Distribution of quantitative variables was analyzed graphically and by the Shapiro-Wilk normality test. Comparison between quantitative variables was performed by analysis of variance (ANOVA). In cases of significant difference, a specific analysis by Tukey's method (a variant of Student's test) was performed. Comparison between categorical variables was performed by the Chisquare test with 2 degrees of freedom. Non-parametric tests (Wilcoxon) were used in the subgroup analysis. Adjusted odds ratios (ORs) were obtained by means of multivariable linear regression analysis with the number of mature oocytes as the dependent variable. We based the selection criteria for variables on the literature regarding prognostic factors of ovarian response. We tested interaction terms between variables at a significance level of 0.05 . The number of cycles was integrated in the statistical analysis.

A power calculation has been conducted at the beginning of recruitment in order to estimate sufficient sample size (44 cycles per group) to achieve adequate power (80\%) with $5 \%$ type I error.

Informed consent was signed by all the participants, and the study was approved by the local institutional review board (CEERB) under authorization number IRB006477.

\section{Results}

The women's mean age was estimated at $32.7 \pm 4.6$ years old. The average duration of infertility was $3.8 \pm 2.9$ years. Among the 157 patients included, 25.8\% presented with a partner with mild male infertility, $8.4 \%$ had a previous history of unilateral tubal surgery, and $6.5 \%$ of bilateral tubal surgery. All the patients had normal ovulation and normal basal hormonal tests of ovarian reserve. The clinical characteristics of the three groups studied are summarized in table 1 . The mean patients' age and duration of infertility were comparable among groups. Male infertility was observed less frequently in the US group than in the BS and NS groups (10.4\% versus $29.4 \%$ and $32.9 \%$, respectively; $p=0.01$ ), whereas primary infertility was observed more frequently in the control group than in the BS and US groups (40.7\% versus $23.5 \%$ and $12.2 \%$, respectively; $\mathrm{p}=0.002$ ).

The antral follicles count and basal FSH and AMH were comparableamong the groups, while inhibin B was significantly reduced in the BS group $(\mathrm{p}=0.04)$. A history of unilateral tubal repair surgery was less frequently encountered in the NS group (4.4\%) versus $11.8 \%$ in the BS group and $14.8 \%$ in 
the US group. In contrast, a history of bilateral tubal surgery was more frequent in the BS group $(17.7 \%$ versus $6.3 \%$ in the US group and $4.4 \%$ in the NS group).

Two hundred seventy-one stimulation cycles for IVF were performed, with an average of two cycles per patient (Table 2 ). The mean interval between salpingectomy, if any, and ovarian stimulation was estimated at 36 months, ranging from 3 to 156 months. Agonist stimulation protocols were the most frequently used in all the groups. The mean duration of stimulation was comparable among the groups. Peak estradiol levels were significantly reduced in the BS group compared to the US and NS groups (1733.9 versus 1905.6 and 2188.0 $\mathrm{pg} / \mathrm{ml}$, respectively; $\mathrm{p}=0.01$ ). Moreover, the total number of oocytes retrieved was significantly reduced in the BS group, compared to the US and NS groups (7.5 versus 9.5 and 9.8, respectively; $p=0.02$ ). Consequently, the number of oocytes inseminated or microinjected was significantly reduced in the BS group $(\mathrm{p}=0.002)$.

Poor ovarian response was more frequently observed in the BS group than in the US and NS groups $(21.7 \%$ versus $9.5 \%$ and $6.9 \%$, respectively; $\mathrm{p}=0.02$ ). The number of embryos obtained was significantly reduced in the BS group $(p=0.04)$, but the implantation rate, pregnancy rate per cycle and live birth rates were comparable among the groups.

\section{Subgroup analysis of unilateral salpingectomy population}

A subgroup analysis of the US population revealed that the mean number of follicles (10 to $16 \mathrm{~mm}$ ) recruited on the day of rhCG administration was lower on the salpingectomy side compared to the contralateral side (6.8 versus 8.2, respectively; $p=0.007)$. After stratification based on the etiology of salpingectomy (i.e., ectopic pregnancy or hydrosalpinx), we observed a significant decrease on the salpingectomy side compared to the contralateral side in the number of follicles recruited $(p=0.005)$ and in the number of mature follicles $(p=0.03)$ on the day of rhCG administration only when the salpingectomy was performed for hydrosalpinges. Similarly, we observed a significant decrease in the number of oocytes retrieved from the salpingectomy side (mean difference of 2.07; $\mathrm{p}=0.02$ ) when tubal surgery was indicated for hydrosalpinges (Table 3).

\section{Multiple regression analysis}

We included previous covariates, in addition to other relevant confounders, in a linear regression model with the number of oocytes as the outcome variable (Table 4). In the bilateral salpingectomy group, the number of mature oocytes retrieved remained reduced by $2.08(\mathrm{p}=0.01)$, compared to that of the control group. No interaction was noticed

Table 1: Clinical characteristics of the study population $(n=157)$ according to specific groups.

\begin{tabular}{|c|c|c|c|c|}
\hline & Bilateral Salpingectomy & Unilateral Salpingectomy & No Salpingectomy & $\mathbf{p}$ \\
\hline Age (years) & $32.3 \pm 3.4$ & $32.7 \pm 5.1$ & $32.7 \pm 4.5$ & 0.94 \\
\hline Duration of infertility (years) & $3.6 \pm 3.1$ & $3.4 \pm 2.8$ & $4.2 \pm 2.9$ & 0.33 \\
\hline Associated moderate male infertility, n (\%) & $5(29.4 \%)^{a}$ & $5(10.4 \%)^{b}$ & $30(32.9 \%)^{a}$ & 0.01 \\
\hline Primary infertility, n (\%) & $4(23.5 \%)^{a}$ & $6(12.2 \%)^{b}$ & $37(40.7 \%)^{a}$ & 0.002 \\
\hline FSH (IU/L) & $7.2 \pm 2.9$ & $7.0 \pm 2.2$ & $7.0 \pm 1.9$ & 0.89 \\
\hline $\mathrm{AMH}(\mathrm{ng} / \mathrm{mL})$ & $2.6 \pm 1.8$ & $3.1 \pm 2.6$ & $2.9 \pm 2.2$ & 0.52 \\
\hline Inhibin B (pg/mL) & $45.0 \pm 22.7^{a}$ & $56.9 \pm 23.0^{\mathrm{b}}$ & $50.9 \pm 23.6^{b}$ & 0.04 \\
\hline Antral follicle count (AFC) & $11.0 \pm 4.2$ & $11.0 \pm 3.9$ & $12.0 \pm 5.1$ & 0.31 \\
\hline \multicolumn{4}{|l|}{ History of tubal repair surgery } & \multirow{3}{*}{0.04} \\
\hline - unilateral, n (\%) & $2(11.8 \%)^{\mathrm{a}}$ & $7(14.6 \%)^{a}$ & $4(4.4 \%)^{b}$ & \\
\hline - bilateral, n (\%) & $3(17.7 \%)^{a}$ & $3(6.3 \%)^{b}$ & $4(4.4 \%)^{b}$ & \\
\hline
\end{tabular}

Note: Values are shown as means \pm Standard Deviations or number (percentage).

a,bsignificant difference between the groups selected (ANOVA or Chi-square).

Table 2: Comparison of stimulation cycles and outcomes of the study population $(n=157)$ according to specific groups.

\begin{tabular}{|c|c|c|c|c|}
\hline & Bilateral Salpingectomy & Unilateral Salpingectomy & No Salpingectomy & $\mathbf{p}$ \\
\hline Number of stimulation cycles & 47 & 79 & 145 & \\
\hline Interval between surgery and IVF, (months) & $38.6 \pm 46.7$ & $45.1 \pm 39.4$ & $32.8 \pm 30.4$ & 0.18 \\
\hline Fertilization technique: IVF, n (\%) & $24(51.1)$ & $53(67.1)$ & $77(53.5)$ & 0.09 \\
\hline Stimulation protocol: Agonist, n (\%) & $41(87.2 \%)$ & $73(92.4 \%)$ & $134(92.4 \%)$ & 0.37 \\
\hline Duration of stimulation (days) & $10.0 \pm 2.4$ & $10.1 \pm 1.6$ & $10.2 \pm 1.5$ & 0.79 \\
\hline Total dose of FSH used (IU) & $2155.1 \pm 776.7$ & $2230.9 \pm 783.1$ & $2373.3 \pm 696.8$ & 0.16 \\
\hline Peak estradiol level $(\mathrm{pg} / \mathrm{mL})$ * & $1733.9 \pm 989^{a}$ & $1905.6 \pm 1038$ & $2188.0 \pm 1025^{b}$ & 0.01 \\
\hline Follicles > $16 \mathrm{~mm}$ * & $5.0 \pm 2.5$ & $5.7 \pm 3.6$ & $6.1 \pm 4.0$ & 0.34 \\
\hline Total oocytes retrieved & $7.5 \pm 4.5^{\mathrm{a}}$ & $9.5 \pm 5.4$ & $9.8 \pm 4.9^{b}$ & 0.02 \\
\hline Oocytes inseminated or microinjected & $5.9 \pm 4.0^{\mathrm{a}}$ & $8.5 \pm 5.1^{b}$ & $8.8 \pm 5.0^{b}$ & 0.002 \\
\hline Poor ovarian response: $\leq 3$ oocytes, $n(\%)$ & $10(21.7)^{\mathrm{a}}$ & $7(9.5)^{b}$ & $10(6.9)^{\mathrm{b}}$ & 0.02 \\
\hline Number of embryos obtained & $3.5 \pm 3.0^{\mathrm{a}}$ & $5.0 \pm 3.8$ & $5.0 \pm 3.2^{b}$ & 0.04 \\
\hline Number of embryos transferred & $1.7 \pm 1.1$ & $1.7 \pm 1.1$ & $1.8 \pm 1.0$ & 0.60 \\
\hline Pregnancy rate per cycle & $8(17.8)$ & $22(29.7)$ & $45(31.3)$ & 0.20 \\
\hline Live birth rate & $3(6.7)$ & $14(19.2)$ & $22(15.3)$ & 0.30 \\
\hline
\end{tabular}

*on the day of hCG administration; a,bsignificant difference between the groups selected (ANOVA or Chi-square). 
Table 3: Comparison of ovarian response on the operated side and the non-operated side in the Unilateral Salpingectomy group, according to the etiology of salpingectomy.

\begin{tabular}{|c|c|c|c|c|c|c|c|c|}
\hline \multirow[t]{2}{*}{ Unilateral Salpingectomy group } & \multicolumn{4}{|c|}{ Salpingectomy for ectopic pregnancy $n=49(62 \%)$} & \multicolumn{4}{|c|}{ Salpingectomy for hydrosalpinges $n=\mathbf{3 0}(\mathbf{3 8} \%)$} \\
\hline & Salpingectomy side & Contra-lateral side & $\Delta$ & $\mathbf{p}$ & Salpingectomy side & Contra-lateral side & $\Delta$ & $\mathbf{p}$ \\
\hline Day 3 AFC* & $5.7 \pm 2.9$ & $5.7 \pm 3.2$ & -0.03 & 0.6 & $5.1 \pm 1.8$ & $5.6 \pm 1.4$ & -0.52 & 0.6 \\
\hline Follicles $(10-16 \mathrm{~mm})^{\star \star}$ & $6.9 \pm 3.7$ & $7.8 \pm 3.9$ & -0.85 & 0.19 & $6.6 \pm 3.3$ & $9.0 \pm 3.8$ & -2.38 & 0.005 \\
\hline 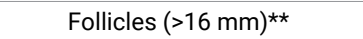 & $2.9 \pm 2.5$ & $2.6 \pm 2.2$ & +0.24 & 0.59 & $2.3 \pm 2.0$ & $3.7 \pm 2.4$ & -1.39 & 0.03 \\
\hline Oocytes retrieved & $4.7 \pm 3.2$ & $4.9 \pm 3.7$ & -0.18 & 0.71 & $3.1 \pm 2.8$ & $5.2 \pm 2.5$ & -2.07 & 0.02 \\
\hline
\end{tabular}

*Antral follicle count, **on the day of hCG administration.

Table 4: Multiple regression model for the number of mature oocytes retrieved $\left(N=238 ; R^{2}=18 \%\right)$.

\begin{tabular}{|c|c|c|c|}
\hline \multirow{2}{*}{ Variable } & \multicolumn{3}{|c|}{ Number of mature oocytes retrieved } \\
\hline & $\beta$ coefficient* & $95 \%$ confidence interval & p value \\
\hline No salpingectomy (ref**) & 0.00 & - & - \\
\hline Unilateral salpingectomy & -0.77 & -2.07 to 0.52 & 0.24 \\
\hline Bilateral salpingectomy & -2.08 & -3.71 to 0.46 & 0.01 \\
\hline
\end{tabular}

* Adjusted for woman's age, interval between surgery and stimulation, etiology of salpingectomy, previous abdominal surgeries, type of infertility, ovarian reserve, stimulation protocol and duration, and total FSH dose used.

** Reference class.

between the etiology of salpingectomy and interval between salpingectomy and ovarian stimulation.

\section{Discussion}

This study showed a significant decrease in peak estradiol levels, in the number of oocytes retrieved and fertilized, and in the number of embryos obtained after BS, compared to the US and NS groups. Poor ovarian response was also more frequent in the BS group despite comparable mean basal characteristics. These results suggest an alteration in ovarian response after bilateral salpingectomy. A decrease in the number of follicles recruited was also observed from the ovary on which salpingectomy was performed, compared to the contralateral side in the US group, which highlights that salpingectomy could be a causal factor in this alteration in ovarian response. In addition, the absence of a history of ovarian surgery in the studied population reinforces the suspected role of salpingectomy.

However, the non-randomized design of this study might have caused some limitations regarding the consistency of the selected groups. In addition to some differences in surgical histories and types of infertility, starting doses of FSH were selected according to the patients' ovarian reserve (antral follicle count and AMH levels). However, the duration of stimulation and total dose of FSH used were comparable among groups, and primary infertility was more frequent in the control group.

Data from the literature regarding this subject have been conflicting. Several studies have not found a significant decrease in ovarian response after salpingectomy [17,18,20-23]. However, most of these studies did not differentiate between unilateral and bilateral salpingectomy and did not mention whether previous adnexal surgeries were performed, and few studies have included large sample sizes, have been sufficiently well-designed, and have discussed salpingectomy indications; Meng, et al. [16] found a significant decrease in the number of oocytes retrieved and significant increases in the dose of gonadotropins and in the duration of stimulation in patients who underwent bilateral salpingectomy, compared to patients without salpingectomy. Furthermore, the authors observed a significant decrease in the number of oocytes retrieved on the salpingectomy side, compared to the contralateral side. We found similar results and further quantified the effects of bilateral salpingectomy on the reduction of mature oocytes at retrieval. This alteration observed in ovarian response after salpingectomy is rather quantitative than qualitative as pregnancy and live birth rates did not differ between groups.

In the subgroup analysis of unilateral salpingectomy, the etiology of salpingectomy was of the utmost importance because ovarian response was significantly reduced after surgery for hydrosalpinges, but this was not the case after surgery for ectopic pregnancies. These results suggest that salpingectomy for hydrosalpinges is potentially more harmful than salpingectomy for ectopic pregnancy regarding future ovarian response to hyperstimulation. Even though we did not have the exact etiology of hydrosalpinx, several hypotheses mightexplain these findings. In ectopic pregnancy situations, the mesosalpinx usually presents important neovascularization, increasing the arterial blood supply not only to the tube but also to the ovary. This neovascularization would protect against the potential damage induced by salpingectomy. In contrast, chronic pelvic inflammatory disease, which is a frequent source of hydrosalpinges, would also affect the ovary through adhesions and/or blood supply alteration. These effects might be emphasized by salpingectomy, thus decreasing ovarian response to stimulation.

Therefore, indications for bilateral salpingectomy for hydrosalpinges should be carefully considered. Indeed, surgical treatment of hydrosalpinges is recommended prior to IVF, especially when hydrosalpinges are visible on ultrasound [10], but salpingectomy should be only bilateral if the hydrosalpinges are bilateral [11]. To improve pregnancy rates in patients with hydrosalpinges without risking a decrease in ovarian response, alternatives to salpingectomy were proposed, such as neosalpingostomy and proximal tubal occlusion, yielding similar IVF outcomes [11,19,24]. 
Many studies have attributed the decrease in ovarian response after salpingectomy to surgical damage to the ovarian blood supply in the mesosalpinx [25-28]. However, this observation might not only be the result of injury to the tubal arcade. Because of numerous anatomical variations in the tubo-ovarian arterial network, the entire ovarian blood supply could be injured after salpingectomy [29]. In fact, Doppler studies have shown a decrease in ovarian blood flow after salpingectomy [13]. Therefore, a minimally invasive surgery is crucial, specifically avoiding cauterization with old generation energies that have strong lateral thermic effects. Data from recent reviews highlighted this potential impair in ovarian response to hyperstimulation during IVF [30], even though it appears that salpingectomy is unlikely to have a substantial adverse impact on pregnancy outcome [31].

In conclusion, bilateral salpingectomy could have a negative effect on ovarian response, when compared with unilateral US or no surgery. It is rather a quantitative effect, limited to 2.1 mature oocytes. It is most likely "etiology-dependent" effect, mainly observed after salpingectomy for hydrosalpinges than for ectopic pregnancy. Good tubal scoring for prognosis could help in making surgical decisions, and alternatives to salpingectomy should be discussed in cases of altered ovarian reserve or if technical difficulties are encountered. Ultimately, even if unilateral salpingectomy does not seem to have a significant effect on ovarian response, it remains to be demonstrated whether this lack of difference is due to a real contralateral offsetting effect or to the potential adjustment of ovarian stimulation by the clinician.

\section{Ethics}

Subjects have given their written informed consent.

The study protocol has been approved by the research institute's committee on human research.

\section{Author's contributions}

CY: statistical analysis, patient recruitment, revision; $\mathrm{AB}$ : revision and writing; CC: data collection and writing; ALM: patient recruitment; SF: patient recruitment

\section{References}

1. Dubuisson JB, Morice P, Chapron C, De Gayffier A, Mouelhi T. Salpingectomy - the laparoscopic surgical choice for ectopic pregnancy. Hum Reprod. 1996; 11: 1199-1203.

PubMed: https://www.ncbi.nlm.nih.gov/pubmed/8671423

2. Andersen AN, Yue Z, Meng FJ, Petersen K. Low implantation rate after in-vitro fertilization in patients with hydrosalpinges diagnosed by ultrasonography. Hum Reprod. 1994; 9: 1935-1938.

PubMed: https://www.ncbi.nlm.nih.gov/pubmed/7844229

3. Dechaud H. [[Hydrosalpinx and fertilization in vitro-embryo transfer: abstention or salpingectomy? Salpingectomy, yes but when and for whom?]. Gynecol Obstet Fertil. 2001; 29: 473-474.

PubMed: https://www.ncbi.nlm.nih.gov/pubmed/11462968

4. Katz E, Akman MA, Damewood MD, Garcia JE. Deleterious effect of the presence of hydrosalpinx on implantation and pregnancy rates with in vitro fertilization. Fertil Steril. 1996; 66: 122-125.

PubMed: https://www.ncbi.nlm.nih.gov/pubmed/8752622

5. Strandell A, Waldenstrom U, Nilsson L, Hamberger L. Hydrosalpinx reduces in-vitro fertilization/embryo transfer pregnancy rates. Hum Reprod. 1994; 9: 861-863.

PubMed: https://www.ncbi.nlm.nih.gov/pubmed/7929732

6. Vandromme J, Chasse E, Lejeune B, Van Rysselberge M, Delvigne A, et al. Hydrosalpinges in in-vitro fertilization: an unfavourable prognostic feature. Hum Reprod. 1995; 10: 576-579.

PubMed: https://www.ncbi.nlm.nih.gov/pubmed/7782435

7. Camus E1, Poncelet C, Goffinet F, Wainer B, Merlet F, et al. Pregnancy rates after in-vitro fertilization in cases of tubal infertility with and without hydrosalpinx: a meta-analysis of published comparative studies. Hum Reprod. 1999; 14: 1243-1249.

PubMed: https://www.ncbi.nlm.nih.gov/pubmed/10325271

8. Zeyneloglu HB, Arici A, Olive DL. Adverse effects of hydrosalpinx on pregnancy rates after in vitro fertilization-embryo transfer. Fertil Steril. 1998; 70: 492-499.

PubMed: https://www.ncbi.nlm.nih.gov/pubmed/9757878

9. Bredkjaer HE1, Ziebe S, Hamid B, Zhou Y, Loft A, et al. Delivery rates after in-vitro fertilization following bilateral salpingectomy due to hydrosalpinges: a case control study. Hum Reprod. 1999; 14: 101-105. PubMed: https://www.ncbi.nlm.nih.gov/pubmed/10374103

10. Strandell A, Lindhard A, Waldenstrom U, Thorburn J, Janson PO, et al. Hydrosalpinx and IVF outcome: a prospective, randomized multicentre trial in Scandinavia on salpingectomy prior to IVF. Hum Reprod. 1999; 14: 2762-2769.

PubMed: https://www.ncbi.nlm.nih.gov/pubmed/10548619

11. Johnson N, van Voorst S, Sowter MC, Strandell A, Mol BW. Surgical treatment for tubal disease in women due to undergo in vitro fertilisation. Cochrane Database Syst Rev. 2010; CD002125. PubMed: https://www.ncbi.nlm.nih.gov/pubmed/15266464

12. Johnson NP, Mak W, Sowter MC. Laparoscopic salpingectomy for women with hydrosalpinges enhances the success of IVF: a Cochrane review. Hum Reprod. 2002; 17: 543-548.

PubMed: https://www.ncbi.nlm.nih.gov/pubmed/11870100

13. Chan $\mathrm{CC}, \mathrm{Ng} \mathrm{EH}, \mathrm{Li} \mathrm{CF}$, Ho PC. Impaired ovarian blood flow and reduced antral follicle count following laparoscopic salpingectomy for ectopic pregnancy. Hum Reprod. 2003; 18: 2175-2180.

PubMed: https://www.ncbi.nlm.nih.gov/pubmed/14507841

14. Gelbaya TA, Nardo LG, Fitzgerald CT, Horne G, Brison DR, et al. Ovarian response to gonadotropins after laparoscopic salpingectomy or the division of fallopian tubes for hydrosalpinges. Fertil Steril. 2006; 85: 1464-1468.

PubMed: https://www.ncbi.nlm.nih.gov/pubmed/16580673

15. Lass A, Ellenbogen A, Croucher C, Trew G, Margara R, et al. Effect of salpingectomy on ovarian response to superovulation in an in vitro fertilization-embryo transfer program. Fertil Steril. 1998; 70: 1035-1038. PubMed: https://www.ncbi.nlm.nih.gov/pubmed/9848291

16. Meng $\mathrm{XH}$, Zhu YM. [Effect of salpingectomy on ovarian function] Zhejiang Da Xue Xue Bao Yi Xue Ban. 2006; 35: 555-559. PubMed: https://www.ncbi.nlm.nih.gov/pubmed/17063552

17. Almog B1, Wagman I, BibiG, RazY, Azem F, etal. Effects of salpingectomy on ovarian response in controlled ovarian hyperstimulation for in vitro fertilization: a reappraisal. Fertil Steril. 2011; 95: 2474-2476. PubMed: https://www.ncbi.nlm.nih.gov/pubmed/21474129

18. Dar P, Sachs GS, Strassburger D, Bukovsky I, Arieli S. Ovarian function before and after salpingectomy in artificial reproductive technology 
patients. Hum Reprod. 2000; 15: 142-144.

PubMed: https://www.ncbi.nlm.nih.gov/pubmed/10611204

19. Kontoravdis A, Makrakis E, Pantos K, Botsis D, Deligeoroglou E, et al. Proximal tubal occlusion and salpingectomy result in similar improvement in in vitro fertilization outcome in patients with hydrosalpinx. Fertil Steril. 2006; 86: 1642-1649.

PubMed: https://www.ncbi.nlm.nih.gov/pubmed/17069813

20. Strandell A, Lindhard A, Waldenstrom U, Thorburn J. Prophylactic salpingectomy does not impair the ovarian response in IVF treatment. Hum Reprod. 2001; 16: 1135-1139.

PubMed: https://www.ncbi.nlm.nih.gov/pubmed/11387282

21. SurreyES,SchoolcraftWB. Laparoscopic management of hydrosalpinges before in vitro fertilization-embryo transfer: salpingectomy versus proximal tubal occlusion. Fertil Steril. 2001; 75: 612-617.

PubMed: https://www.ncbi.nlm.nih.gov/pubmed/11239550

22. Tal J, Paltieli Y, Korobotchka R, Ziskind G, Eibschitz I, et al. Ovarian response to gonadotropin stimulation in repeated IVF cycles after unilateral salpingectomy. J Assist Reprod Genet. 2002; 19: 451-455. PubMed: https://www.ncbi.nlm.nih.gov/pubmed/12416648

23. Verhulst G, Vandersteen N, van Steirteghem AC, Devroey P. Bilatera salpingectomy does not compromise ovarian stimulation in an in-vitro fertilization/embryotransfer programme. Hum Reprod. 1994; 9: 624-628. PubMed: https://www.ncbi.nlm.nih.gov/pubmed/8046013

24. Ducarme G, Uzan M, Hugues JN, Cedrin-Durnerin I, Poncelet C Management of hydrosalpinx before or during in vitro fertilizationembryo transfer: a national postal survey in France. Fertil Steril. 2006;
86: 1013-1016.

PubMed: https://www.ncbi.nlm.nih.gov/pubmed/16962113

25. Alvarez F, Faundes A, Brache V, Tejada AS, Segal S. Prospective study of the pituitary-ovarian function after tubal sterilization by the Pomeroy or Uchida techniques. Fertil Steril. 1989; 51: 604-608.

PubMed: https://www.ncbi.nlm.nih.gov/pubmed/2494079

26. Cattanach J. Oestrogen deficiency after tubal ligation. Lancet. 1985; 1 : 847-849.

PubMed: https://www.ncbi.nlm.nih.gov/pubmed/2858712

27. Cattanach JF, Milne BJ. Post-tubal sterilization problems correlated with ovarian steroidogenesis. Contraception. 1988; 38: 541-550. PubMed: https://www.ncbi.nlm.nih.gov/pubmed/3197418

28. Radwanska E, Headley SK, Dmowski P. Evaluation of ovarian function after tubal sterilization. J Reprod Med. 1982; 27: 376-384. PubMed: https://www.ncbi.nlm.nih.gov/pubmed/6811740

29. Chauvin C, Koskas M, Yazbeck C. [Salpingectomy--how I do it]. Gynecol Obstet Fertil. 2010; 38: 776-777.

PubMed: https://www.ncbi.nlm.nih.gov/pubmed/21030283

30. Fan M, Ma L. Effect of salpingectomy on ovarian response to hyperstimulation during in vitro fertilization: a meta-analysis; Fertil Steril. 2016; 106: 322-329.

PubMed: https://www.ncbi.nlm.nih.gov/pubmed/27114330

31. Kotlyar A, Gingold J, Shue S, Falcone T. The Effect of salpingectomy on ovarian function. J Minim Invasive Gynecol. 2017; 24: 563-578. PubMed: https://www.ncbi.nlm.nih.gov/pubmed/28223181 